\title{
Remoción de partículas adsorbentes de iones cobre por flotación $\operatorname{Jet}^{(\bullet)}$
}

\author{
M. Santander*, P. Tapia*, O. Pávez*, L. Valderrama*y D. Guzmán* \\ Resumen \\ Los resultados que se presentan en este artículo corresponden a los estudios realizados para la remoción de iones co- \\ bre desde efluentes sintéticos, utilizando la técnica de flotación por partículas adsorbentes (FPA) en una celda de \\ flotación Jet (tipo Jameson). En los estudios de adsorción y flotación se utilizó, como partículas adsorbentes, mineral \\ con alto contenido de cuarzo, para determinar condiciones óptimas de $\mathrm{pH}$, concentración de partículas adsorben- \\ tes, dosis óptimas de reactivos de flotación, razón flujo de aire/flujo de efluente necesaria de aplicar en la celda Jet, pa- \\ ra maximizar la eficiencia de adsorción de iones cobre y remoción de partículas adsorbentes de iones cobre. Los resul- \\ tados indican que, a pH > 7 y concentraciones de partículas adsorbentes de $2 \mathrm{~kg} \cdot \mathrm{m}^{-3}$, se adsorbe el $99 \%$ de los iones \\ cobre y cuando la razón flujo de aire/flujo de efluente, aplicada en la celda Jet, es 0,2, se remueve un 98 \% de las par- \\ tículas adsorbentes de iones cobre.
}

Palabras clave

Flotación Jet; Adsorción; Eliminación de $\mathrm{Cu}^{+2}$.

\section{Removal of adsorbent particles of copper ions by Jet flotation}

\begin{abstract}
The present study shows the results obtained on the removal of copper ions from synthetic effluents by using the adsorbent particles flotation technique (APF) in a Jet flotation cell (Jameson type). In a typical experimental run, a mineral with high quartz content was used as adsorbent particles in the adsorption and flotation experiments, to determine optimal $\mathrm{pH}$ conditions, adsorbent particles concentration; flotation reagents dosage and air/effluent flow ratio for applying in the Jet cell to maximize the efficiency of copper ions adsorptions and the removal of particles adsorbent containing the absorbed copper ions. The results indicate that at $\mathrm{pH}>7$ and at adsorbent particles concentration of $2 \mathrm{~kg} \cdot \mathrm{m}^{-3}, 99 \%$ of copper ions is adsorbed and, when the air/ effluent flow ratio applied in the Jet cell is 0.2 , $98 \%$ of adsorbent particles containing the adsorbed copper ions is removed.
\end{abstract}

Keywords

Jet flotation; Adsorption; Elimination of $\mathrm{Cu}^{+2}$.

\section{INTRODUCCIÓN}

Entre las técnicas descritas en la literatura para la descontaminación de efluentes o residuos industriales líquidos (RIL's) contaminados con iones de metales pesados generados en diferentes procesos industriales, se encuentra la adsorción combinada con flotación, conocida como técnica de flotación por partículas adsorbentes (FPA).

Como partículas adsorbentes de iones de metales pesados se han utilizado materiales inorgánicos de origen mineral, tales como zeolitas, bentonita, caolín, diatomea, pirita, cuarzo, etc. ${ }^{[1-10]}$.

La técnica FPA ha sido aplicada para remover, desde efluentes líquidos, diferentes iones de metales pesados. Zouboulis et al. ${ }^{[10]}$ realizaron experiencias, a escala de laboratorio, para la remoción de $\mathrm{Cu}^{+2}$ y $\mathrm{Cr}^{+6}$ desde soluciones acuosas, utilizando partículas finas de pirita (pureza $95 \%$ y tamaño medio $12 \mu \mathrm{m}$ ) y flotación por aire disperso para la separación sólido/líquido. En estos estudios se analizaron; influencia del pH, concentración inicial del ión de metal pesado, concentración de pirita, tipo y concentración de reactivo colector e influencia del tiempo de contacto. Los resultados alcanzados por estos investigadores, les permitió concluir que la técnica FPA para la remoción de iones de metales pesados es un proceso efectivo, pues lograron la remoción completa, en las condiciones óptimas. Para el caso de remoción de iones cromo, la pirita presenta la ventaja de

(•) Trabajo recibido el día 28 de octubre de 2008 y aceptado en su forma final el día 13 de abril de 2009.

* Universidad de Atacama -Departamento de Metalurgia, Centro Regional de Investigación y Desarrollo Sustentable de Atacama, Copiapó, Chile. mario.santander@uda.cl 
actuar como un agente reductor del cromo hexavalente a trivalente.

Rubio y Tessele ${ }^{[1]}$, obtuvieron resultados de remoción de iones cinc, cobre y níquel, desde soluciones diluidas utilizando zeolitas $(100 \%<74 \mu \mathrm{m})$ como partículas adsorbentes y flotación por aire disuelto para la separación sólido/líquido. Los resultados presentados por estos autores demostraron que es posible remover alrededor de un $98 \%$ de los iones de metales pesados, utilizando $\mathrm{Fe}(\mathrm{OH})_{3}$, como coagulante de las partículas adsorbentes. Féris et al. ${ }^{[11]}$ aplicaron la técnica FPA a la remoción de iones metálicos $(\mathrm{Cu}, \mathrm{Zn}$, $\mathrm{Ni}, \mathrm{Cd}, \mathrm{Cr}$ y Ag) en efluentes metalúrgicos, en escala piloto, utilizando un subproducto del beneficio de carbón como partículas adsorbentes. En la operación de separación sólido/líquido usaron equipos de flotación por aire disuelto y columnas de flotación. Los resultados alcanzados por dichos autores, demostraron que ambos procesos, FPA-FAD y FPA-Columna, son eficientes para tratamiento de efluentes que contienen iones metálicos; sin embargo, la FAD presenta índices de remoción superior a los de la columna.

Zouboulis et al. ${ }^{[12]}$ investigaron la remoción de iones zinc y cromo desde soluciones acuosas utilizando goetita $(\alpha-\mathrm{FeO}(\mathrm{OH}))$, como partículas adsorbentes. La etapa de separación sólido/líquido la realizaron en columnas de flotación de laboratorio y estudiaron la alternativa de utilizar colectores producidos biológicamente (Surfactin-105 y Lichenysin-A), en reemplazo del colector producido químicamente (dodecilsulfato de sodio). Los resultados obtenidos por estos investigadores los llevó a concluir que los colectores producidos por métodos biológicos son más efectivos para remover $\mathrm{Cr}^{+6}$ después de la adsorción en la goetita, que el colector dodedilsulfato de sodio. Sin embargo, el colector Lichenysin-A no resultó efectivo para la remoción de zinc.

El equipo de flotación ampliamente empleado en el tratamiento de efluentes líquido es el de flotación por aire disuelto (FAD), debido a que los contaminantes contenidos en estos residuos, ya sean éstos iones de metales pesados (precipitados, adsorbidos en coloides adsorbentes o en partículas adsorbentes), sólidos en suspensión o partículas líquidas coaguladas y/o floculadas, etc., son frágiles y de tamaño pequeño. Para evitar que éstos se rompan y se puedan remover de manera eficiente es necesario utilizar equipos de flotación que generen un gran número de burbujas de tamaño pequeño y condiciones hidrodinámicas de bajo cizalle. La FAD cumple con esta condición ya que utiliza micro burbujas (burbujas de 30 a $100 \mu \mathrm{m}$ de diámetro), sin embargo tiene baja capacidad de tratamiento del orden de $4-15 \mathrm{~m}^{3} / \mathrm{m}^{2} \mathrm{~h}^{[13}$ y 14]. Actualmente, existen equipos de flotación con mayor capacidad de tratamiento, que la FAD, en los cuales las dos funciones básicas de la flotación (formación de los agregados partícula-burbujas de aire y la separación posterior de estos agregados desde el líquido) se realizan en dos reactores separados. Este modo de operación los torna adecuados para ser utilizados en el tratamiento de efluentes líquidos. Entre los equipos basados en este concepto, están la celda Jameson y las celdas neumáticas ${ }^{[15]}$. La principal aplicación a nivel industrial de estas celdas es en la concentración de minerales, sin embargo, su utilización se ha extendido al tratamiento de efluentes líquidos y a la purificación de electrolitos de soluciones de extracción por solvente ${ }^{[14,16 \text { y } 17]}$.

El objetivo de este estudio es demostrar la aplicabilidad de las Celdas de Flotación Jet (CFJ) para la remoción de iones cobre, adsorbidos en un mineral con alto contenido de cuarzo.

\section{DESARROLLO EXPERIMENTAL}

\subsection{Materiales y equipos}

El efluente sintético con una concentración de 0,02 $\mathrm{kg} \cdot \mathrm{m}^{-3}$ de $\mathrm{Cu}^{2+}$ y a $\mathrm{pH} 2$ se preparó disolviendo $\mathrm{CuSO}_{4} \times 5 \mathrm{H}_{2} \mathrm{O}$, p.a. de $99 \%$ de pureza, marca Fluka, en agua potable. $\mathrm{El} \mathrm{pH}$ fue regulado, según la necesidad de cada estudio, con hidróxido de sodio o ácido clorhídrico de calidad analítica, marca Riedel, y registrado con un $\mathrm{pH}$-metro HANNA HI 8424. Como reactivos colectores se utilizó lauril sulfato de sodio, p.a, marca Sudelab y oleato de sodio, p.a, marca Riedel, y como espumante se usó metil isobutil carbinol-MIBC. Para la preparación de los reactivos se utilizó agua desmineralizada. La concentración de cobre en el efluente sintético, antes y después de los estudios de adsorción y flotación, se determinó en un espectrofotómetro de absorción atómica (GBC 908).

Las partículas adsorbentes utilizadas corresponde a desmontes (mineral con baja ley de hierro) colectados en yacimientos de minerales de hierro, ubicados en la Región de Atacama-Chile. La muestra fue reducida de tamaño 100 \%-200 mallas ASTM (100 \%-74 $\mu \mathrm{m})$, homogeneizada y cuarteada, a fin de obtener muestras representativas para su caracterización y para los ensayos de adsorción y flotación. La determinación de las especies cristalinas presentes en la muestra se realizó por difracción de rayos x (DRX), en un Difractómetro, marca Bruker AXS, modelo D8 Advance, mientras que la composición química se determinó por fluorescencia de rayos x (FRX) en un Espectrómetro SIEMENS SRS 3000. El área superficial y diámetro de poros se calculó mediante la adsorción de nitrógeno, según el método de BET, en el analizador, Modelo QS-7 Quantachrome Co., Greenvale, NY. La caracterización morfológica de la muestra se 
llevó a cabo en un microscopio electrónico de barrido (SEM), marca LEO, modelo 420.

Los estudios de adsorción se realizaron en un vaso precipitado de $1 \mathrm{dm}^{3}$ con 4 baffles y un agitador mecánico, marca "Stuart Scientific modelo SS3", de velocidad variable.

Los estudios de flotación FPA fueron realizados en una Celda de Flotación Jet (CFJ). La figura 1a) muestra una representación esquemática de la celda y los equipos auxiliares. Esta celda está compuesta por un tubo de descenso (reactor), un tanque de flotación (separador) y un sistema de control de nivel. Los equipos auxiliares incluyen un acondicionador de $0,05 \mathrm{~m}^{3}$, una bomba helicoidal de flujo variable, marca Moyno, y un flujometro. El tubo de descenso tiene en el extremo superior un inyector para la generación de burbujas, (Fig. 1 b)) y en el extremo inferior un difusor (Fig. $1 \mathrm{c}$ )).

El efluente es bombeado desde el acondicionador a través del inyector que tiene en su interior una boquilla de $3 \mathrm{~mm}$ de diámetro; la pared de la boquilla es de un material poroso. El paso del efluente en alta velocidad por la boquilla, crea un vacío, induciendo la entrada de aire, autoaspirado o inducido, por un orificio lateral dispuesto para este fin en la pared externa del inyector. El aire es forzado a pasar a través de los poros de la pared de la boquilla, lo que da lugar a la formación de las burbujas en la pared interna de la boquilla; mientras mayor sea la velocidad de paso del líquido por la boquilla, más rápido se desprenderán las burbujas y menor será su tamaño. Las burbujas generadas en el inyector, son forzadas a descender por el tubo de descenso en sentido opuesto a su flotabilidad natural, en dirección al tanque de flotación. En esta celda, el proceso de "colección" (colisión-adhesión), formación de los agregados partículas/burbujas de aire, se produce en el tubo de descenso (reactor) y la separación de los agregados desde el agua se produce en el tanque de flotación (separador). La mezcla cae en co-corriente, siendo descargada por el difusor en dirección ascendente en el tanque de flotación. Este modo de descarga evita que las partículas que se desprenden de las burbujas sean arrastradas junto con el efluente clarificado, ya que aumenta la probabilidad de que sean colectadas por un nuevo flujo ascendente de burbujas. Los agregados

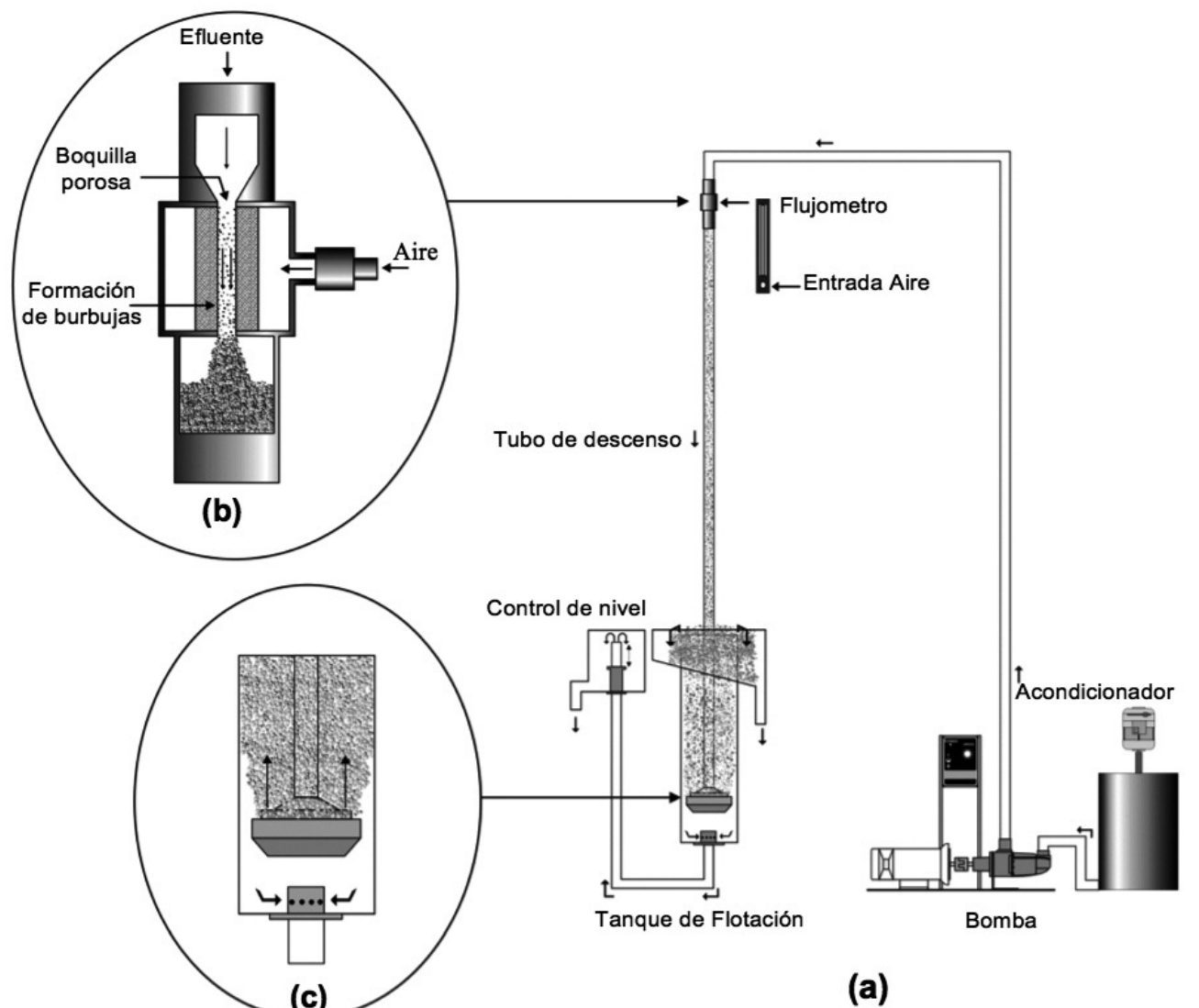

Figura 1. (a) Representación esquemática de la celda de flotación jet y sus equipos auxiliares, (b) Inyector y (c) Difusor.

Figure 1. (a) Schematic representation of the jet flotation cell and its auxiliary equipments, (b) Injector and (c) Diffuser. 
ascienden a la superficie (camada de espuma), mientras que el agua tratada se descarga por el fondo del tanque de flotación. La altura de la camada de espuma y la descarga desde el tanque de flotación es regulada por el sistema de control de nivel.

\subsection{Metodologías}

\subsubsection{Estudios de adsorción}

Los ensayos de adsorción se ejecutaron con 0,6 dm de efluente sintético contaminado con $0,02 \mathrm{~kg} \cdot \mathrm{m}^{-3}$ de iones cobre, concentraciones de 2 y $10 \mathrm{~kg} \cdot \mathrm{m}^{-3} \mathrm{de}$ partículas adsorbentes, a temperatura ambiente, a una velocidad de agitación de $1.500 \mathrm{rpm}$ y tiempo de adsorción de $600 \mathrm{~s}$. El pH se ajustó después de la adición de las partículas adsorbentes en los valores predefinidos para cada ensayo, entre 2 y 12 .

Una vez finalizada la etapa de adsorción se separó el sólido del líquido mediante filtración al vacío utilizando papel Filtro Whatman con tamaño de poros $5 \mu \mathrm{m}$. En el líquido filtrado se midió el $\mathrm{pH}$ y se colectó una muestra para determinar la concentración residual de iones cobre. La muestra se preservó acidificándola con $\mathrm{HNO}_{3} 50$ \%. La adsorción de iones cobre en las partículas adsorbentes fue determinada por la diferencia entre la concentración de los iones analizados en la muestra de efluente sintético colectada antes de la adsorción y la colectada después de la adsorción.

\subsubsection{Estudios de flotación}

Previamente a los estudios de remoción de las partículas adsorbentes de iones cobre en la CFJ, se realizaron ensayos preliminares en una columna de flotación batch a fin de establecer las dosis óptimas de colector y espumante que permitieran remover alrededor de un $98 \%$ de las partículas adsorbentes. Se seleccionó como colector primario lauril sulfato de sodio (LSS) y como colector secundario para reforzar la acción colectora del LSS, se utilizó oleato de sodio, ambos colectores son utilizados para oxi-minerales, y como espumante se usó metil isobutil carbinol. Se realizaron pruebas variando la dosis LSS entre 0,2 y 0,01 $\mathrm{kg} \cdot \mathrm{m}^{-3}$ y la dosis de oleato de sodio entre 0 y 0,01 $\mathrm{kg} \cdot \mathrm{m}^{-3}$. La dosis de espumante se fijó en 0,005 kg. $\mathrm{m}^{-3}$.

Todos los ensayos de flotación, en la CFJ, se realizaron con $0,05 \mathrm{~m}^{3}$ de efluente sintético. La etapa de adsorción de iones cobre en las partículas adsorbentes se realizó en el acondicionador que alimenta a la $\mathrm{CFJ}$, pues dispone de un agitador mecánico de velocidad variable. Una vez finalizada la etapa de adsor- ción se adicionó colector y espumante, en las dosificaciones óptimas determinadas en los ensayos preliminares y se acondicionó durante 300 s. Después que las partículas adsorbentes fueron hidrofobizadas el efluente se alimentó a la CFJ. Una vez alcanzadas las condiciones de operación estables, 180 s después de iniciado el ensayo, se colectaron muestras del efluente clarificado. Las muestras fueron acidificadas con $\mathrm{HNO}_{3} 50 \%$ y analizadas por adsorción atómica. La remoción de las partículas adsorbentes de iones cobre se determinó por la diferencia entre la concentración de iones cobre en la muestra colectada en el flujo de alimentación a la celda de flotación y la muestra colectada en el flujo de efluente clarificado.

\section{RESULTADOS Y DISCUSIÓN}

\subsection{Caracterización de las partículas adsorbentes}

El análisis químico por FRX realizado a las partículas adsorbentes, presentado en la tabla 1, muestra que los elementos más abundantes son oxígeno con $48,22 \%$, seguido de silicio con $31,49 \%$ y hierro con $12,23 \%$. En el difractograma DRX, (Fig. 2), se observa que las únicas especies cristalinas presentes en

Tabla I. Análisis químico por fluorescencia de rayos $\mathrm{X}$ de la muestra de partículas adsorbentes

Table I. Chemical analyses for X-ray fluorescence of the sample of adsorbent particles

\section{Elemento Concentración, \% peso}

$\begin{array}{cr}\mathrm{O} & 48,22 \\ \mathrm{Si} & 31,49 \\ \mathrm{Fe} & 12,23 \\ \mathrm{Al} & 3,80 \\ \mathrm{Ti} & 1,45 \\ \mathrm{~S} & 1,32 \\ \mathrm{Na} & 0,61 \\ \mathrm{P} & 0,32 \\ \mathrm{Ca} & 0,21 \\ \mathrm{Cl} & 0,11 \\ \mathrm{~K} & 0,10 \\ \mathrm{Sr} & 0,07 \\ \mathrm{Cr} & 0,02 \\ \mathrm{Cu} & 0,02 \\ \mathrm{Zr} & 0,02\end{array}$




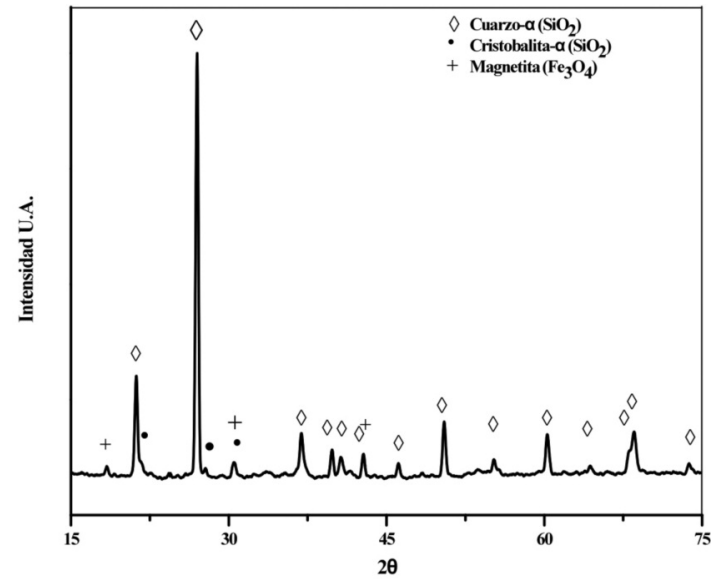

Figura 2. Análisis de difracción de rayos $x$ de la muestra de partículas adsorbentes.

Figure 2. X-ray difraction pattern of the adsorbent particles sample.

la muestra de partículas adsorbentes son cuarzo- $\alpha$ cristobalita- $\alpha$ y magnetita, pero la intensidad de los pick demuestra que el cuarzo- $\alpha$ es la especie cristalina predominante.

La figura 3, a) y b), muestra las micrografías, con las características morfológicas, de las partículas adsorbentes, obtenidas por microscopia electrónica de barrido (SEM). En la figura 3 a), se observa que cada partícula es un agregado de partículas más pequeñas y que cada uno de estos agregados tiene forma geométrica regular, esférica o elipsoidal y tamaño homogéneo del orden de $100 \mu \mathrm{m}$. En la figura $3 \mathrm{~b}$ ), vista magnificada, se observa que las partículas que forman los agregados tienen tamaño inferior a $2 \mu \mathrm{m}$.

El área superficial específica BET de las partículas adsorbente es $13,52 \pm 0.022 \mathrm{~m}^{2} \cdot \mathrm{g}^{-1}$ y el diámetro promedio de poros es $6,54 \mathrm{~nm}$. Este valor del área superficial es demasiado elevada considerando que la especie cristalina principal es cuarzo- $\alpha$. Tikhomolova y Urakova ${ }^{[18]}$ informan de valores de área superficial del orden de $0,20-0,21 \mathrm{~m}^{2} \cdot \mathrm{g}^{-1}$ para partícula de cuarzo- $\alpha$ con tamaño entre 40-70 um y 99,8 \% de pureza.

Las características morfológicas de las partículas (cada partícula esta formada por agregados de partículas con tamaño menor que $2 \mu \mathrm{m}$ ) y la posible presencia de una especie amorfa de hierro, limonita $(\mathrm{FeO}(\mathrm{OH}))$, podrían justificar el elevado valor en el área superficial. La presencia de una especie amorfa de hierro puede tener explicación en el origen de la muestra de partículas adsorbentes, desmontes de minerales de hierro, y en los análisis de DRX y FRX. Según el análisis DRX la única especie cristalina que contiene hierro es la magnetita, sin embargo, la intensidad de su pick es baja y el porcentaje en peso de hierro en la muestra, según el análisis FRX, es significativo, 12,23\%.

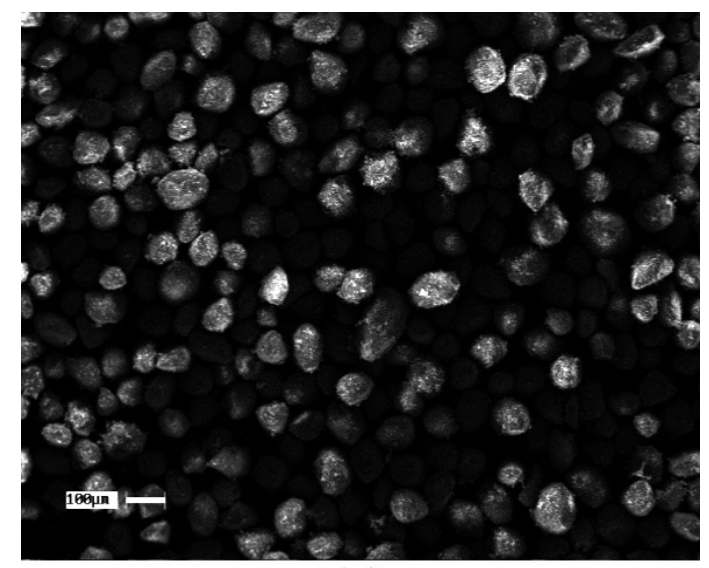

(a)

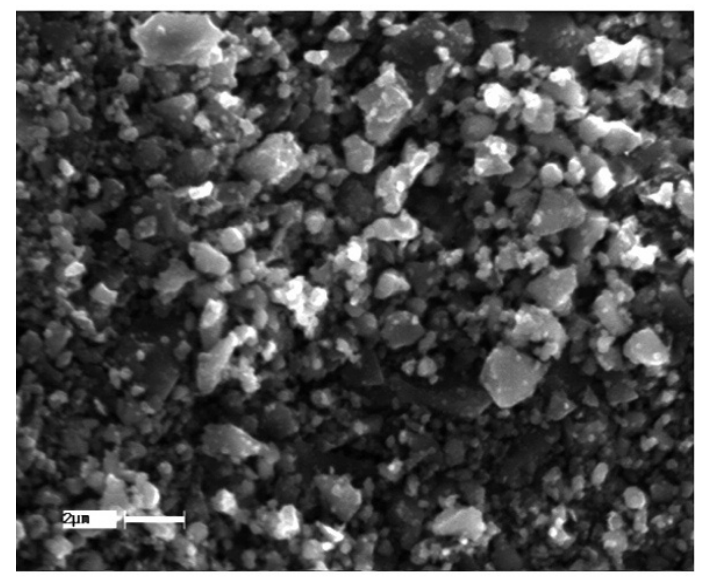

(b)

Figura 3. Micrografías SEM de la muestra de partículas adsorbentes: (a) vista general y (b) vista magnificada.

Figure 3. SEM micrograph of the adsorbent particles sample: (a) general view and (b) magnified view.

\subsection{Estudios de adsorción}

\subsubsection{Efecto del $p H$}

La figura 4 presenta los resultados de los ensayos de adsorción y precipitación (ensayo blanco) de iones cobre. En la figura, se observa que el $\mathrm{pH}$ afecta fuertemente la adsorción y precipitación de iones cobre. En ambos casos, cuando el pH fue modificado, desde la zona ácida a la básica, el porcentaje de iones cobre en solución disminuye, sin embargo, la curva que representa la adsorción está más desplazada hacia la zona de $\mathrm{pH}$ ácido, que la curva que representa la precitación. Para los mismos valores de $\mathrm{pH}$ el porcentaje de iones cobre en solución es menor en los ensayos de adsorción que en los ensayos de precipitación. Los ensayos 


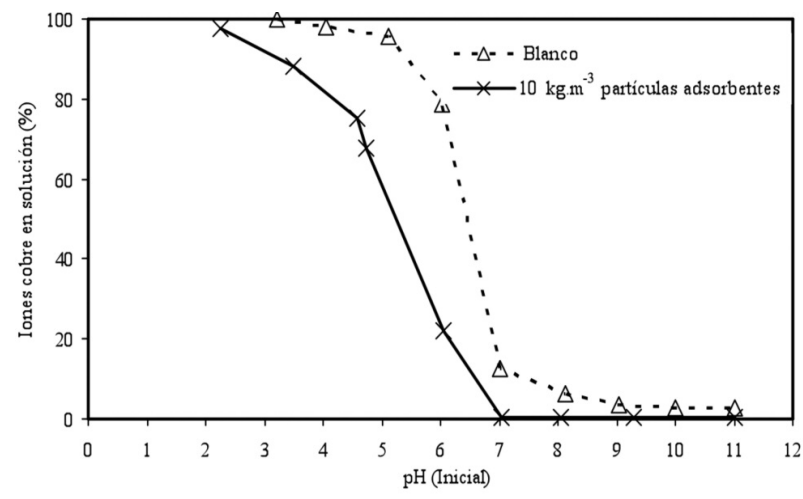

Figura 4. Efecto del pH sobre el porcentaje de iones cobre en solución para los ensayos precipitación y adsorción de iones cobre.

Figure 4. The Effect of $\mathrm{pH}$ on the percentage of copper ions in solution for testing precipitation and adsorption of copper ions.

de adsorción muestran que a $\mathrm{pH} 5$ el porcentaje de iones cobre en solución es del orden de $60 \%$ (40\% de los iones cobre son adsorbidos) y a $\mathrm{pH} 7$ es del orden de $1 \%$ (99\% de los iones cobre son adsorbidos). Sin embargo los ensayos de precipitación muestran que a pH 5 el porcentaje de iones cobre en solución es del orden de $95 \%$ y a pH 7 es del orden de $15 \%$.

Resultados similares del efecto del $\mathrm{pH}$ sobre la adsorción de iones cobre en materiales con alto contenido de sílice y oxihidróxidos de hierro han sido expuestos por diferentes investigadores ${ }^{[19}$ y 24$]$. La mayoría de las investigaciones coinciden en que la adsorción en este tipo de materiales es influenciada, intensamente, por el $\mathrm{pH}$ ya que éste determina el grado de ionización y las especies de cobre en solución, y la carga superficial del adsorbente.

El diagrama de distribución de especies de cobre en función del pH exhibido en la figura 5, se graficó para una concentración $0,02 \mathrm{~kg} \cdot \mathrm{m}^{-3}$ utilizando las constante de equilibrio de las reacciones de hidrólisis de $\mathrm{Cu}^{+2}$ reportados por Weng et al. ${ }^{[25]}$. El diagrama indica que a $\mathrm{pH}<5,5$, la especie iónica predominante es $\mathrm{Cu}^{+2}$, a pH en el rango de 5,5 a 10 las especies iónicas predominantes son $\mathrm{Cu}^{+2}, \mathrm{Cu}(\mathrm{OH})^{+}$y $\mathrm{Cu}(\mathrm{OH})_{2}$, y a $\mathrm{pH}>10$ las especies iónicas dominantes son $\mathrm{Cu}(\mathrm{OH})_{2}$ y $\mathrm{Cu}(\mathrm{OH})_{3}^{-}$. Mediciones de potencial $z$ realizadas por diferentes investigadores revelan que la carga superficial de las partículas de sílice a $\mathrm{pH}>2,8$ es negativa ${ }^{[22}$ y 28$]$.

Larson y Pugh ${ }^{[29]}$ afirmaron que no existe un consenso entre los diferentes investigadores sobre la especie exacta de metal involucrada en el proceso de adsorción. Las diferentes posibilidades incluyen iones no hidrolizados, $\mathrm{Cu}^{+2}$, el primer producto de hidrólisis,

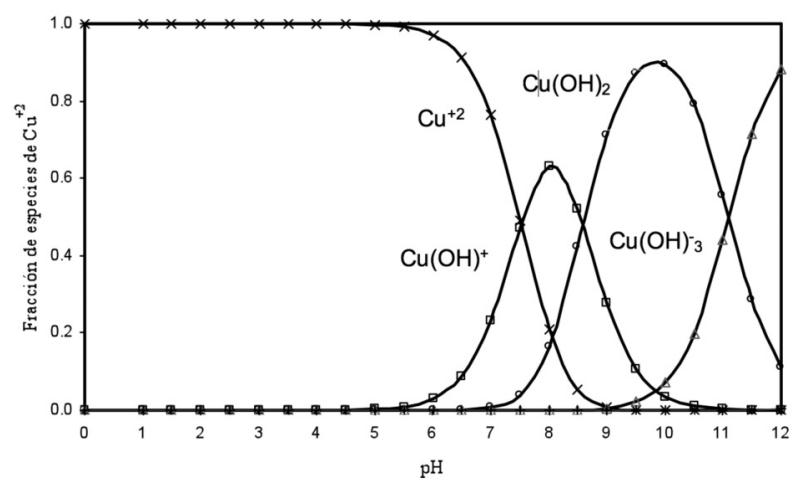

Figura 5. Distribución de especies de $\mathrm{Cu}^{+2}$ en función del $\mathrm{pH}$ para una concentración inicial de $0,02 \mathrm{~kg} \cdot \mathrm{m}^{-3}$, graficado utilizando las constantes de equilibrio reportadas en la referencia ${ }^{[25]}$.

Figure 5. Distribution of $\mathrm{Cu}^{+2}$ species as function of $\mathrm{pH}$ for an initial concentration $0.02 \mathrm{~kg} . \mathrm{m}^{-3}$, calculate using the equilibrium constant report on reference ${ }^{[25]}$.

$\mathrm{Cu}(\mathrm{OH})^{+}$y dímeros $\mathrm{Cu}(\mathrm{OH})_{2}^{z^{+}}$. Alinnor ${ }^{[20]}$ y Cho et al. ${ }^{[22]}$, informaron que los complejos de hidróxido de metales tienen mayor afinidad para adsorberse que los metales hidratados, debido a que el grupo $\mathrm{OH}$ reduce la energía requerida para la adsorción.

Diversos estudios explican los resultados experimentales de la adsorción de iones $\mathrm{Cu}^{+2}$ en la interfase agua/sílice, en términos de formación de complejos superficiales con grupos silanoles como ligandos $[18,26$, 27,30 y 31 . Por otro lado, investigaciones realizadas utilizando técnicas de espectroscopía de absorción de rayos X en capa fina extendida (EXFS) revelaron que los iones $\mathrm{Cu}^{+2}$ forman diferentes complejos superficiales sobre óxidos y oxihidróxidos de hierro, dependiendo del $\mathrm{pH}$ y fuerza iónica. Según Bochatay et al. ${ }^{[24]}$, sobre oxihidróxidos, los iones $\mathrm{Cu}^{+2}$ interactúan directamente con el hierro sin que una molécula de agua este presente, en una estructura llamada complejo superficial de esfera interna. Peacock y Sherman ${ }^{[23]}$ utilizando esta misma técnica, identificaron la formación de complejos superficiales bidentados y tridentados entre iones $\mathrm{Cu}^{+2}$, óxidos y oxihidróxidos de hierro.

Considerando la naturaleza heterogénea del mineral utilizado como partículas adsorbentes los mecanismos de adsorción deben ser la suma de interacciones físicas y químicas entre los sitios superficiales del adsorbente y los iones $\mathrm{Cu}^{+2}$. A pH $<7$ el mecanismos más probable es la adsorción química específica de los iones $\mathrm{Cu}^{+2}$ con los grupos superficiales del adsorbente, principalmente, vía formación de complejos, y a pH $>7$ el mecanismo de adsorción más factible es la precipitación de $\mathrm{Cu}(\mathrm{OH})_{2}$ sobre la superficie del adsorbente. 


\subsubsection{Efecto de la concentración de partículas adsorbentes}

En la figura 6 se presentan los resultados de los ensayos realizados para analizar el efecto de la concentración de partículas adsorbentes. En la figura, se observa que, a $\mathrm{pH}>5$, el porcentaje de adsorción de iones cobre es independiente de la concentración de partículas adsorbentes y, a $\mathrm{pH}<5$, el porcentaje de adsorción aumenta cuando la concentración de partículas adsorbentes se incrementa, o sea, la curva de adsorción se desplaza hacia la zona de $\mathrm{pH}$ ácido. En pH 4, se obtienen porcentajes de adsorción del orden de $18 \%$ cuando se utilizó $10 \mathrm{~kg} \cdot \mathrm{m}^{-3}$ de partículas adsorbentes y, en este mismo valor de $\mathrm{pH}$, se obtuvo un porcentaje de adsorción del orden de $5 \%$, cuando se utilizó $2 \mathrm{~kg} \cdot \mathrm{m}^{-3}$ de partículas adsorbentes. Cho et al. ${ }^{[22]}$, también estudiaron el efecto de la concentración de partículas adsorbentes (cenizas con 57,82\% $\mathrm{SiO}_{2}$ ) sobre la remoción de varios metales y encontraron un efecto contrario al observado en este estudio: a pH en el rango de 3 a 5 el porcentaje de cobre adsorbido es independiente de la dosis de adsorbente; a $\mathrm{pH}>5$ la curva de adsorción de iones cobre se desplaza a la zona de $\mathrm{pH}$ ácido, es decir, el porcentaje de adsorción de iones cobre aumenta cuando la concentración de partículas adsorbentes se incrementa. Estudios realizados por Bouzid et al. ${ }^{[19]}$ demostraron que un incremento en la concentración de partículas adsorbentes, aumenta la adsorción de iones cobre en el rango de $\mathrm{pH} 3$ a 7.

Con base en los resultados alcanzados en los estudios de adsorción se puede afirmar que los desmontes de minerales de hierro tienen potencial para ser utilizados en la remoción de iones de metales pesados desde

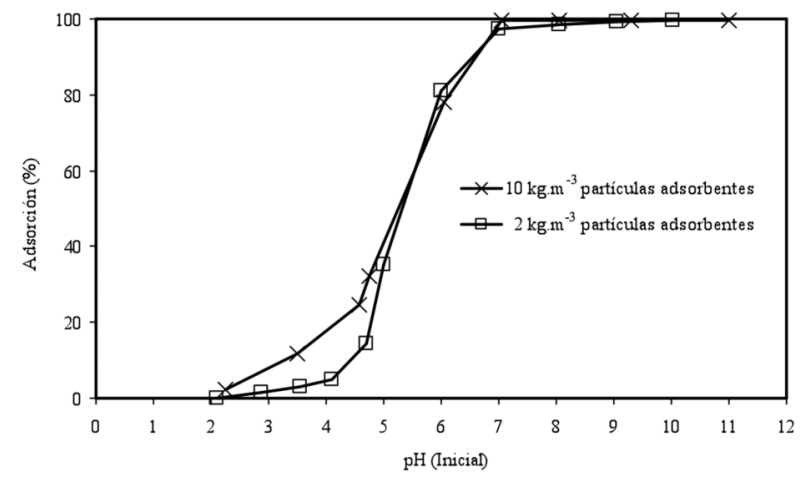

Figura 6. Efecto del pH sobre porcentaje de adsorción de iones cobre para concentraciones de partículas adsorbentes de $2 \mathrm{~kg} \cdot \mathrm{m}^{-3}$ y $10 \mathrm{~kg} \cdot \mathrm{m}^{-3}$

Figure 6. The effect of $\mathrm{pH}$ on the adsorption of copper ions at adsorbent particles concentrations of $2 \mathrm{~kg} \cdot \mathrm{m}^{-3}$ and $10 \mathrm{~kg} \cdot \mathrm{m}^{-3}$. efluentes líquidos, generados en operaciones mineras y metalúrgicas. Las principales ventajas de este adsorbente son: que es un desecho de bajo costo, que es posible eliminarlo del efluente por flotación y que está disponible en grandes cantidades en las faenas mineras donde puede ser utilizado.

\subsection{Estudios de flotación}

\subsubsection{Ensayos preliminares}

En la tabla II se presentan los valores de las dosis óptimas del colector que permitieron remover un $98 \%$ de las partículas adsorbentes. Se seleccionó una concentración de $0,1 \mathrm{~kg} \cdot \mathrm{m}^{-3}$ de lauril sulfato de sodio y 0,002 $\mathrm{kg} \cdot \mathrm{m}^{-3}$ de oleato de sodio, a pesar de que con dosis mayores de oleato de sodio se alcanzaron porcentajes de remoción de partículas adsorbentes de, aproximadamente $99 \%$, sin embargo, por inspección visual, se observó que el efluente tratado presentaba un color lechoso debido al exceso de reactivos.

También se realizaron ensayos preliminares para verificar la estabilidad de la CFJ, utilizando las dosis óptimas de colector y espumante presentadas en la tabla II y los valores de variables de operación exhibidos en la tabla III. Mediante inspección visual, se observó que para flujos de efluente mayores que $0,108 \mathrm{dm}^{3} \cdot \mathrm{s}^{-1}$, el régimen de flujo del tubo descenso se tornó inestable,

Tabla II. Dosis de reactivo utilizados en los estudios de flotación en la celda Jet

Table II. Reagents dosage used in the Jet cell flotation studies

\begin{tabular}{lc}
\hline \multicolumn{1}{c}{ Reactivo } & Dosis, $\mathbf{k g} \cdot \mathbf{m}^{\mathbf{3}}$ \\
\hline Oleato de sodio & 0,002 \\
Lauril sulfato de sodio & 0,10 \\
MIBC & 0,005 \\
\hline
\end{tabular}

Tabla III. Valores de la variables de operación utilizados en los estudios de flotación en la celda Jet

Table III. Variable operation values used in the Jet cell flotation studies

\begin{tabular}{ll}
\hline \multicolumn{1}{c}{ Variable } & Flujo, $\mathbf{d m}^{3} \cdot \mathbf{s}^{-1}$ \\
\hline Flujo de aire & $0,0083-0,0500$ \\
Flujo de efluente & $0,0366-0,1166$ \\
\hline
\end{tabular}


probablemente, debido a que al aumentar el flujo de efluente se generó, en la parte superior del tubo de descenso, una zona de flujo turbulento, sumado a esto, también, se observó que, a medida que la mezcla de aire y efluente descendían por el tubo de descenso, las burbujas aumentaron de tamaño por efecto de coalescencia. Lo anterior trae como consecuencia que la mezcla de agregados partículas adsorbentes/burbujas se descargaran, con mayor velocidad, en el tanque de separación de las fases, generando turbulencia en la parte superior de éste. Según Cowburn et al. ${ }^{[32]}$, un aumento de la velocidad superficial del jet de líquido en la celda Jameson requiere, sustancialmente, más energía con el consiguiente aumento de la turbulencia. Para disminuir la coalescencia de las burbujas en el tubo de descenso y aumentar la estabilidad de la capa de espuma, se incremento la dosis de espumante a $0,017 \mathrm{~kg} \cdot \mathrm{m}^{-3}$.

\subsubsection{Efecto del flujo de efluente}

En la figura 7 se presentan los resultados de los ensayos realizados para analizar la influencia de flujo de efluente, alimentado a la CFJ, sobre el porcentaje de remoción de partículas adsorbentes de iones cobre. Dichos ensayos se ejecutaron con las dosis de reactivos definidas en los ensayos preliminares: el flujo de aire se fijó en $0,0166 \mathrm{dm}^{3} \cdot \mathrm{s}^{-1}$ y el $\mathrm{pH}$ en 7,5. En la figura, se observa que un aumento en el flujo de efluente en el rango de 0,0614 a $0,1020 \mathrm{dm}^{3} \cdot \mathrm{s}^{-1}$, produjo un pequeño incremento en el porcentaje de remoción de partículas adsorbentes. Para un flujo de efluente de $0,0614 \mathrm{dm}^{3} \cdot \mathrm{s}^{-1}$, la eficiencia o porcentaje de remoción de partículas adsorbentes de iones cobre es del orden

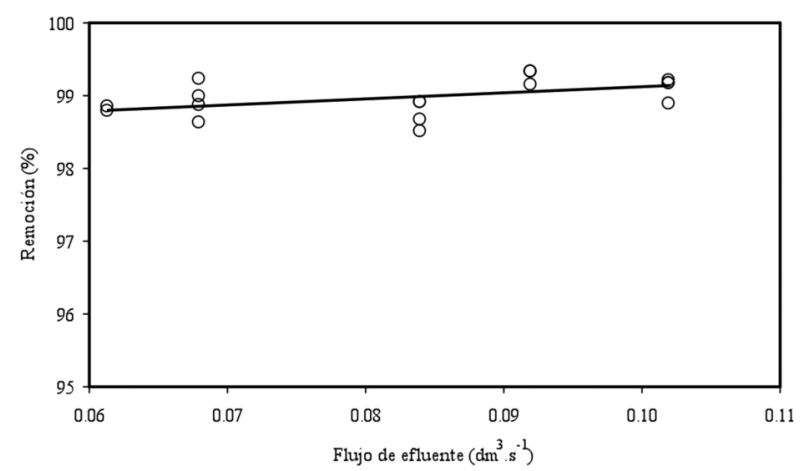

Figura 7. Efecto de flujo de efluente alimentado a la celda de flotación Jet sobre el porcentaje de remoción de partículas adsorbentes de iones cobre.

Figure 7. The effect of effluent flow rate fed to the jet flotation cell on the removal percentage of the adsorbent particles the copper ions. de $98,81 \%$ y para un flujo de efluente de 0,1020 $\mathrm{dm}^{3} \cdot \mathrm{s}^{-1}$, es del orden de $99,10 \%$.

Un incremento en el flujo de efluente disminuyó el tiempo de retención en el tubo de descenso y en el tanque de flotación, según se observa en la figura 8. Un aumento del flujo de alimentación disminuye el tamaño de las burbujas y aumenta la velocidad de evacuación del producto flotado. Una disminución del tamaño de las burbujas incrementa el número de éstas y su área superficial, por lo tanto, se acrecienta la probabilidad de formación de agregados partículas adsorbentes-burbujas de aire y, consecuentemente, aumenta el porcentaje de remoción de partículas adsorbentes de iones cobre. Un incremento en la velocidad de evacuación de concentrado, también contribuye a acrecentar el porcentaje de remoción de partículas adsorbentes de iones cobre, debido a que disminuye el espesor de la capa de espuma y la probabilidad de rompimiento de los agregados partículas adsorbentes-burbujas de aire.

Estudios realizados en la celda Jameson demostraron que un incremento en la velocidad del flujo de concentrado aumenta la recuperación de partículas de cuarzo, debido a una disminución del espesor de la capa de espuma ${ }^{[33]}$. Diferentes investigadores concuerdan en que un aumento en la altura de espuma disminuye la

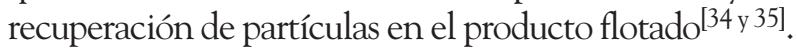

\subsubsection{Efecto de la razón flujo de aire/ flujo de efluente}

La figura 9 muestra los resultados del efecto de la razón flujo de aire/flujo de efluente sobre el porcentaje de remoción de partículas adsorbentes. En estos ensayos,

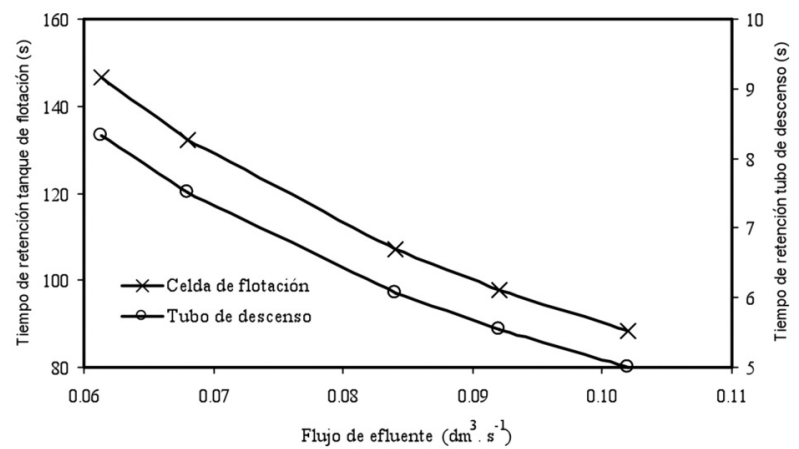

Figura 8. Efecto de flujo de efluente alimentado a la celda de flotación Jet sobre tiempo de retención en el tubo de descenso y tanque de flotación.

Figure 8. The effect of effluent flow rate fed to the jet flotation cell on the retention time in the descent tube and the flotation tank. 


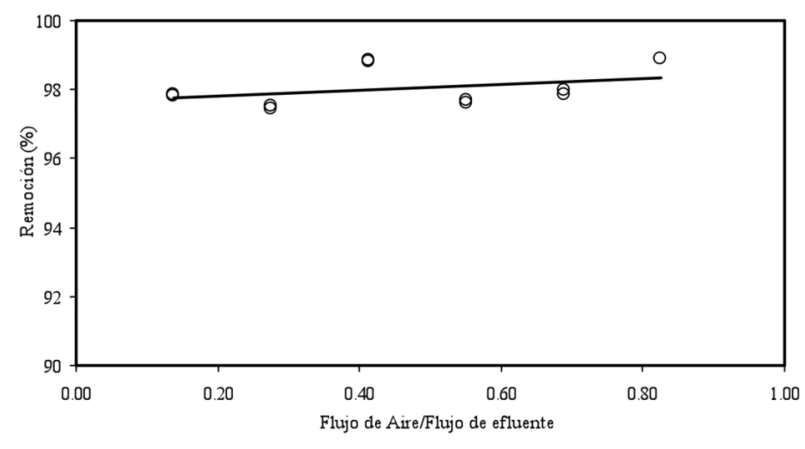

Figura 9. Efecto de la razón flujo de aire/ flujo de efluente alimentado a la celda de flotación Jet sobre el porcentaje de remoción de partículas adsorbentes de iones cobre.

Figure 9. The effect of the ratio between the air flow and the effluent flow fed to jet flotation cell on the removal percentage of the adsorbent particles of copperions.

se utilizó la misma dosis de reactivos definidas en los ensayos preliminares; se fijó el flujo de efluente en 0,0614 $\mathrm{dm}^{3} \cdot \mathrm{s}^{-1}$, el $\mathrm{pH}$ en 7,5 y flujo de aire se modificó en el rango de 0,00833 a $0,0500 \mathrm{dm}^{3} \cdot \mathrm{s}^{-1}$.

En la figura 8 se observa que un aumento en la razón flujo de aire/flujo de efluente, también, produce un pequeño incremento en el porcentaje de remoción de partículas adsorbentes de iones cobre. El porcentaje de remoción se incrementó desde 97,41 \% a 98,90\%, cuando dicha razón aumenta de 0,14 a 0,83. Este incremento en el porcentaje de remoción se debe, probablemente, a que cuando se aumentó el flujo de aire y se mantuvo constante el flujo de efluente, se incrementó la concentración de burbujas en el tubo de descenso (holdup de aire) y, como consecuencia de esto se incrementó la probabilidad de formación de agregado partículas adsorbentes-burbujas de aire.

El holdup de aire es una variable que controla el área superficial de las burbujas y es afectado por varias variables de diseño y operación de la celda Jameson ${ }^{[36-39]}$. Estudios realizados por Tasdemir ${ }^{[33]}$, demostraron que un incremento en el holdup de aire en el tubo de descenso incrementó la recuperación de partículas de cuarzo con tamaño menor a $53 \mu \mathrm{m}$.

\subsubsection{Efecto del pH}

La influencia del $\mathrm{pH}$ sobre el proceso combinado (adsorción y flotación) fue estudiada bajo las siguientes condiciones experimentales: $\mathrm{pH} 7,5$ y 5,2, razones flujo de aire/ flujo de efluente variando en el rango 0,2 a 0,9 y las mismas dosis de reactivos de flotación utilizadas en los ensayos preliminares. Los resultados están representados en la figura 10 e indican que el porcentaje de remoción de partículas adsorbentes de iones cobre es menor en $\mathrm{pH}$ 5,2 que en $\mathrm{pH} 7,5$. Sin embargo, el porcentaje de remoción en ambos valores de $\mathrm{pH}$ coincide con el porcentaje de adsorción. En pH 5,2, el porcentaje iones cobre en solución (Fig. 4) es del orden de $55 \%$ (45 \% de adsorción) y el porcentaje de remoción (Fig. 10) es del orden de $46 \%$. De forma análoga, en pH 7,5, el porcentaje iones cobre en solución, (Fig. 4), es del orden de $1 \%$ (99 \% de adsorción) y el porcentaje de remoción (Fig. 10) es del orden de $98 \%$.

La CFJ debido a su elevada eficiencia de remoción de partículas adsorbentes de iones cobres, a su elevada velocidad de flotación, del orden de $45 \mathrm{~m}^{3} / \mathrm{m}^{2} \mathrm{~h}$, a su facilidad para operar con altas concentraciones de sólidos en suspensión y a su bajo consumo de energía debido a que no necesita comprimir aire a alta presión para generar las burbujas, tiene un alto potencial para ser utilizada en el tratamiento de efluentes líquidos contaminados con iones de metales pesado y sólidos en suspensión.

\section{CONCLUSIONES}

Los resultados obtenidos permiten concluir que la técnica de flotación por partículas adsorbentes, utilizando un mineral con alto contenido de cuarzo como adsorbente de iones cobre y la celda de flotación Jet, presenta potencial aplicación para el tratamiento de efluente contaminado con iones de metales pesados. En las mejores condiciones de operación, es posible remover,

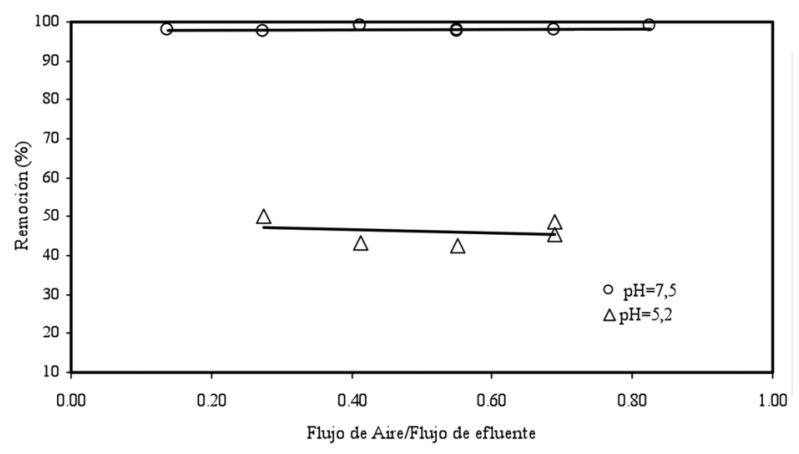

Figura 10. Efecto de la razón flujo de aire/ flujo de efluente alimentado a la celda de flotación Jet sobre el porcentaje de remoción de partículas adsorbentes de iones cobre en pH 7,5 y 5,2.

Figure 10. The effect of the ratio between the air flow and the effluent flow fed to jet flotation cell on the removal percentage of the adsorbent particles of copper ions at pH 7.5 and 5.2. 
desde un efluente contaminados con $0,02 \mathrm{~kg} \cdot \mathrm{m}^{-3}$ de $\mathrm{Cu}^{+2}$, un $98 \%$ de las partículas adsorbentes de iones cobre.

La adsorción de iones cobre está fuertemente influenciada por el $\mathrm{pH}$. La cantidad de $\mathrm{Cu}^{+2}$ adsorbido se incrementa con el $\mathrm{pH}$, alcanzándose porcentajes de adsorción del orden de $99 \%$, a $\mathrm{pH}>7$. A $\mathrm{pH}>5$, el porcentaje de adsorción de $\mathrm{Cu}^{+2}$ es independiente de la concentración de partículas adsorbentes, alcanzándose los mismos porcentajes de adsorción, cuando se emplearon concentraciones de partículas adsorbentes de 2 y $10 \mathrm{~kg} \cdot \mathrm{m}^{-3}$.

Un aumento en el flujo de efluente, en el rango de $0,0633 \mathrm{a} 0,1020 \mathrm{dm}^{3} \cdot \mathrm{s}^{-1}$ y de la razón flujo de aire/flujo de efluentes en el rango 0,14 a 0,83, produce un pequeño incremento en el porcentaje de remoción de partículas adsorbentes de iones cobre.

\section{Agradecimientos}

Los autores agradecen el apoyo financiero aportado por la Dirección de Investigaciones de la Universidad de Atacama a través del financiamiento del proyecto de investigación 221141 DIUDA 06/03. También, agradecen la colaboración del Sr. Bruno Zazzali y Sra. Jenny Patrian por su apoyo en el desarrollo experimental del trabajo.

\section{REFERENCIAS}

[1] J. Rubio y F. Tessele, Miner. Eng. 10 (1997) 671679.

[2] I. Lin y I. Spevakova, Prog. Miner. Process. Technol. 58 (1994) 431-450.

[3] S. Kesraoui-Ouki, C. Cheeseman y R. Perry, J. Chem. Technol. Biotechnol. 59 (1994) 121-126.

[4] M. Zamzow y E. Murphy, Sep. Sci. Technol. 27 (1984) 1969-1984.

[5] G. Pramod, P. Albino, S. Chakrabory y M. Ray, Sep. Purif. Technol. 57 (2007) 47-56.

[6] L. Feris, A. De Leon, M. Santander y J. Rubio, Int. J. Miner. Process. 74 (2004) 101-106.

[7] C. Costa, Disertación Magister, Escola de Engenharia, Programa Pós-Graduaçao em Engeharia Metalúrgica e dos Materiais, Universidad Federal do Rio Grande do Sul, 1998.

[8] K. Keydros, K. Matis y G. Stalidis, J. Coll. Int. Sci. 155 (1993) 409-414.

[9] A. Zouboulis, Miner. Eng. 8 (1995) 1.477-1.488.

[10] A. Zouboulis, K. Kydros y K. Matis, Sep. Sci. Technol. 27 (1992) 2.143-2.155.

[11] L. Férís, M. Souza, J. Rubio, VI Southern Hemisphere Meeting on Mineral Technology, Rio de Janeiro, Brasil, 2001, pp.436-442.
[12] A. Zouboulis, K.Matis, N. Lazaridis y P. Golyshin, Miner. Eng. 16 (2003) 1.231-1.236.

[13] J. Solari, Engenharia Saitaria 20 (1981) 332-335.

[14] J. Rubio, M. Souza y R. Smith, Miner. Eng. 15 (2002) 139-155.

[15] J. Finch, Miner. Eng. 8 (1995) 587-602.

[16] Y. Yan y G. Jameson, Int. J. Miner. Process 73 (2004) 23-28.

[17] D. Readett y B. Clayton, Flotation Plants: Are They Optimized? Society for Mining, Metallurgy and Exploration, Littleton, Malhotra, D. (Ed.), 1995, pp. 165-170.

[18] K. Tikhomolova y I. Urakova, Coll. J. USSR 75 (2002) 894-899.

[19] J. Bouzid, Z. Elouear, M. Ksibi, M. Feki, y A. Montiel, J. Hazard Mater. 152 (2008) 838-845.

[20] I. Alinnor, Fuel 86 (2007) 853-858.

[21] A. Papandreou, C. Stournaras, D. Panias, J. Hazard Mater. 148 (2007) 538-547.

[22] H. Cho, D. Oh, K. Kim, J. Hazard Mater. B127 (2005) 187-195.

[23] C. Peacock y D. Sherman, Geochim. Cosmochim. Acta. 68 (2004) 2623-2637.

[24] L. Bochatay, P. Persson, L. Lovgren y G.Brown, J. Phys. IV 7 (1997) 819-820.

[25] C. Weng, C. Tsai, S. Chu y Y. Sharma, Sep. Purif. Technol. 54 (2007) 187-197.

[26] P. Schindler, B. Furst, R. Dick and P. Wolf, J. Coll. Int. Sci. 55 (1976) 469-475.

[27] K. Panday, G. Prasad y V. Singh, Water Res. 19 (1985) 869-873

[28] D. Fornasiero y J. Ralston, Int. J. Miner. Process. 76 (2005) 75-81.

[29] I. Larson y R. Pugh, J. Coll. Int. Sci. 208 (1998) 399-404.

[30] R. Shawabkeh, A. Al-Harahsheh y A. AlOtoom, Sep. Purif. Technol. 40 (2004) 251-257.

[31] N. Vlasova, Colloids Surf. A: Physicchem. Eng. Aspects 163 (2000) 125-133.

[32] J. Cowburn a, G. Harbort, E. Manlapig, Z. Pokrajcic, Miner. Eng. 19 (2006) 609-618.

[33] A. Tasdemir, T. Tasdemir, B. Oteyaka, Miner. Eng. 20 (2007) 1.331-1.336.

[34] H. Soto, G. Barbery, Miner. Metall. Proc. 8 (1991) 16-21.

[35] B. Oteyaka, H. Soto, Miner. Eng. 8 (1995) 91-100.

[36] J. Jameson, E. Manlapig, Proc. Int. Conf. Column Flotation, Sudbury, Ontario, 1991, pp. 673-687.

[37] T. Tasdemir, B. Oteyaka y A. Tasdemir, Miner. Eng. 20 (2007) 761-765.

[38] M. Marchese, A. Uribe y J. Finch, Chem. Eng. Sci. 47 (1992) 3.475-3.482.

[39] K. Yamagiwa, D. Kusabiraki y A Ohkawa, J. Chem. Eng. Jpn. 23 (1990) 343-348. 